Supporting Information

\title{
Effect of urea as electrolyte additive for stabilization of lithium metal electrodes
}

Hee-Sang Kim, Rakesh Verma, Jaekook Kim, and Chan-Jin Park *

Department of Materials Science and Engineering, Chonnam National University, 77,

Yongbongro, Bukgu, Gwangju 61186, South Korea

${ }^{*}$ Corresponding author.

Tel.: +82-62-530-1704. Fax: +82-62-530-1699.

E-mail address: parkcj@jnu.ac.kr_(C.J.Park)

Number of pages: 11 
Number of tables: 4

Number of figures: 7 
Table S1. Fitted parameters based on equivalent circuit models (Fig.S2) that correspond to impedance of Li symmetric cell with $0.5 \mathrm{M}$ urea additive.

\begin{tabular}{|c|c|c|c|c|c|c|c|}
\hline \multirow[t]{2}{*}{ Sample } & \multirow{2}{*}{$\begin{array}{l}R_{s} \\
(\Omega)\end{array}$} & \multirow{2}{*}{$\begin{array}{l}R_{\text {SEI }} \\
(\Omega)\end{array}$} & \multicolumn{2}{|l|}{$\mathrm{CPE}_{\mathrm{SEI}}$} & \multirow{2}{*}{$\begin{array}{l}\mathrm{R}_{\mathrm{ct}} \\
(\Omega)\end{array}$} & \multicolumn{2}{|l|}{$\mathrm{CPE}_{\mathrm{dl}}$} \\
\hline & & & $C_{S E I}(F)$ & $\mathrm{n}_{\mathrm{SEI}}$ & & $\mathrm{C}_{\mathrm{dl}}(\mathrm{F})$ & $\mathrm{n}_{\mathrm{dl}}$ \\
\hline \multirow[t]{2}{*}{ After $1^{\text {st }}$ cycle } & 7.52 & 1127. & $9.94 \times 10^{-4}$ & 0.638 & 858.7 & $1.03 \times 10^{-5}$ & 0.790 \\
\hline & & 2 & & & & & \\
\hline After $5^{\text {th }}$ cycles & 8.28 & 22.58 & $6.96 \times 10^{-5}$ & 0.770 & 51.0 & $4.39 \times 10^{-6}$ & 0.889 \\
\hline After $10^{\text {th }}$ cycles & 8.28 & 22.56 & $6.97 \times 10^{-5}$ & 0.770 & 51.1 & $4.38 \times 10^{-6}$ & 0.890 \\
\hline After $50^{\text {th }}$ cycles & 8.87 & 16.18 & $2.56 \times 10^{-5}$ & 0.797 & 6.00 & $7.26 \times 10^{-6}$ & 1.021 \\
\hline
\end{tabular}

Table S2. Fitted parameters based on equivalent circuit models (Fig. S2) that correspond to impedance of $\mathrm{Li}$ symmetric cell without $0.5 \mathrm{M}$ urea additive.

\begin{tabular}{|c|c|c|c|c|c|c|c|}
\hline \multirow[t]{2}{*}{ Sample } & \multirow{2}{*}{$\begin{array}{l}R_{s} \\
(\Omega)\end{array}$} & \multirow{2}{*}{$\begin{array}{l}\mathbf{R}_{\mathrm{SEI}} \\
(\Omega)\end{array}$} & \multicolumn{2}{|l|}{$\mathrm{CPE}_{\text {SEI }}$} & \multirow{2}{*}{$\begin{array}{l}R_{c t} \\
(\Omega)\end{array}$} & \multicolumn{2}{|l|}{$\mathrm{CPE}_{\mathrm{dl}}$} \\
\hline & & & $\mathrm{C}_{S E I}(\mathrm{~F})$ & $\mathrm{n}_{\text {SEI }}$ & & $C_{d l}(F)$ & $\mathrm{n}_{\mathrm{dl}}$ \\
\hline After $1^{\text {st }}$ cycle & 10.08 & 19.66 & $3.37 \times 10^{-2}$ & 0.448 & 19.86 & $2.18 \times 10^{-5}$ & 0.829 \\
\hline After $5^{\text {th }}$ cycles & 6.46 & 22.48 & $1.99 \times 10^{-4}$ & 0.545 & 9.60 & $5.92 \times 10^{-2}$ & 0.736 \\
\hline After $10^{\text {th }}$ cycles & 64.62 & 867.0 & $8.59 \times 10^{-6}$ & 0.445 & 834.11 & $2.96 \times 10^{-2}$ & 0.157 \\
\hline
\end{tabular}


Table S3. Binding energies of $C 1 \mathrm{~s}, \mathrm{O} 1 \mathrm{~s} F$ 1s, S2p3/2, and $N$ 1s analogous to the fitted peaks of the Li electrode from the Li symmetric cell.

\begin{tabular}{|c|c|c|c|c|c|}
\hline \multirow[t]{2}{*}{ Components } & \multicolumn{5}{|c|}{ Binding energy $(\mathrm{eV})$} \\
\hline & C1s & 01s & F1s & S2p3/2 & N1s \\
\hline Hydrocarbon & 285 & & & & \\
\hline LiTFSI & 292.6 & & 688.5 & 168.7 & 399.4 \\
\hline $\mathrm{Li}_{2} \mathrm{O}$ & & 528.8 & & & \\
\hline LiF & & & 684.9 & & \\
\hline $\mathrm{Li}_{3} \mathrm{~N}$ & & & & & 397 \\
\hline $\mathrm{Li}_{2} \mathrm{~S}$ & & & & 161.2 & \\
\hline $\mathrm{Li}_{2} \mathrm{~S}_{2} \mathrm{O}_{4}$ & & & & 166.2 & \\
\hline $\mathrm{Li}_{2} \mathrm{SO}_{4}$ & & & & 169.7 & \\
\hline Polysulfur & & & & 163.8 & \\
\hline $\mathrm{Li}_{2} \mathrm{CO}_{3}$ & 290.3 & 531.8 & & & \\
\hline$-\mathrm{COO}$ & $287-288$ & 532.3 & & & \\
\hline$C=O$ & 286.1- & 533.6 & & & \\
\hline & 287.1 & & & & \\
\hline$\left(\mathrm{NH}_{2}\right)_{2} \mathrm{CO}$ & 288.7 & 535.0 & & & 399.5 \\
\hline
\end{tabular}


Table S4. Vibrational frequencies and assignments for bis(trifluoromethane sulfonyl)imide lithium salt (LiTFSI) and urea.

\begin{tabular}{|c|c|c|c|}
\hline LiTFSI & & Urea & \\
\hline $\begin{array}{l}\text { Wavenumbe } \\
r \\
\left(\mathrm{~cm}^{-1}\right)\end{array}$ & $\begin{array}{l}\text { Vibrational } \\
\text { mode }\end{array}$ & $\begin{array}{l}\text { Wavenumbe } \\
r \\
\left(\mathrm{~cm}^{-1}\right)\end{array}$ & $\begin{array}{l}\text { Vibrational } \\
\text { mode }\end{array}$ \\
\hline 2876 & $\vartheta \mathrm{S}-\mathrm{CF}_{3}$ & 3430 & $\vartheta \mathrm{N}-\mathrm{H}$ \\
\hline 1325 & $\vartheta \mathrm{C}^{-\mathrm{SO}_{2}-\mathrm{N}}$ & 3332 & $\vartheta_{\text {as }} \mathrm{NH}_{2}$ \\
\hline 1244 & $\vartheta_{\text {as }} \mathrm{SO}_{2}$ & 3256 & $\vartheta_{\mathrm{s}} \mathrm{NH}_{2}$ \\
\hline 1198 & $\vartheta_{\mathrm{as}} \mathrm{CF}$ & 1675 & $\delta_{\mathrm{s}} \mathrm{NH}_{2}$ \\
\hline 1141 & $\vartheta \mathrm{C}-\mathrm{SO}_{2}-\mathrm{N}$ & 1619 & $\delta_{a s} \mathrm{NH}_{2}$ \\
\hline 1061 & $\vartheta_{\text {as }}$ S-N-S & 1590 & $\vartheta_{\mathrm{S}} \mathrm{C}=\mathrm{O}$ \\
\hline 811 & $\delta_{\mathrm{s}} \mathrm{CF}_{3}$ & 1458 & $\vartheta \mathrm{C}-\mathrm{N}$ \\
\hline 747 & $\vartheta \mathrm{S}-\mathrm{N}$ & & \\
\hline 635 & $\delta_{s} \mathrm{O}-\mathrm{S}-\mathrm{O}$ & & \\
\hline 572 & $\delta_{\text {as }} \mathrm{CF}_{3}$ & & \\
\hline 512 & $\delta_{a s} \mathrm{O}-\mathrm{S}-\mathrm{O}$ & & \\
\hline
\end{tabular}




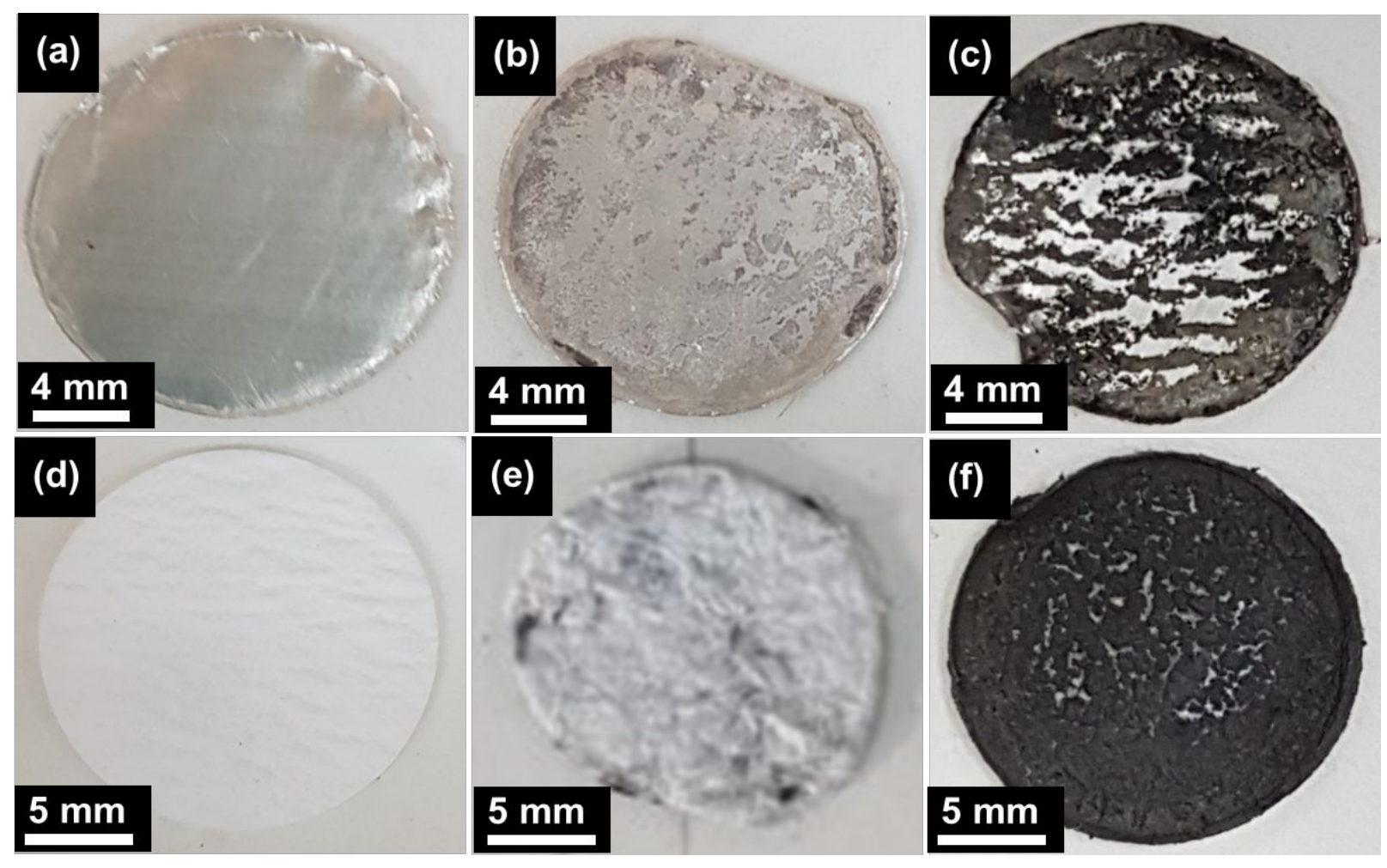

Figure S1. Typical images of components of Li symmetric cells: (a) pristine Li metal; Li

metals from cells (b) with and (c) without $0.5 \mathrm{M}$ urea additive; (d) pristine separator; (e) separator from cells (e) with and (f) without $0.5 \mathrm{M}$ urea additive. The cells were cyclic tested for 10 cycles at current density of $1.0 \mathrm{~mA} \mathrm{~cm}-2$; each discharge-charge cycle lasted $2 \mathrm{~h}$. 


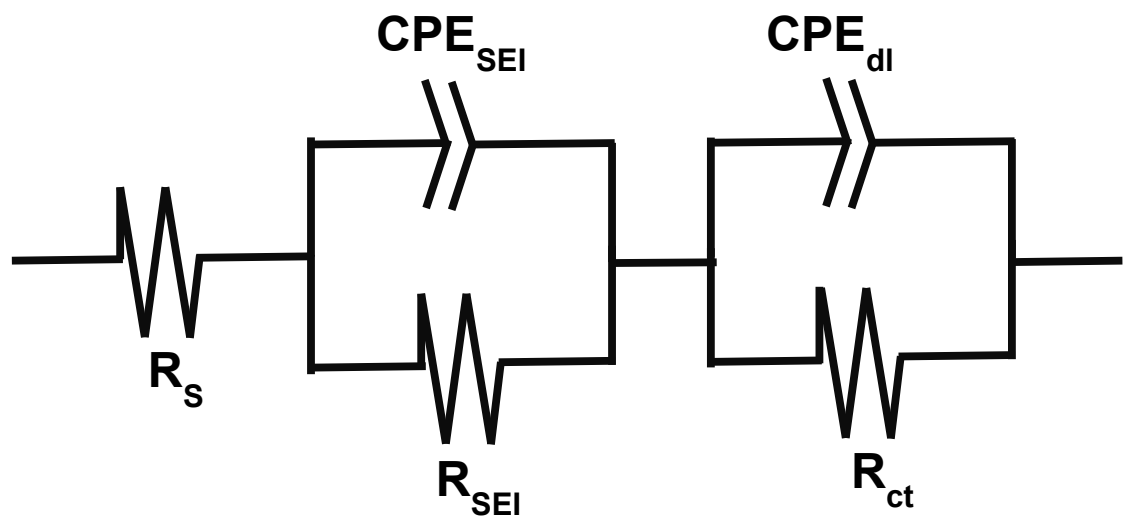

Figure S2. Equivalent circuit for electrochemical impedance spectroscopy curve of Li/Li symmetric cell. Here, $R_{S}, R_{S E l}$, and $R_{c t}$ denote the electrolyte resistance, solid electrolyte interphase (SEI) layer resistance attributed to reaction products found on the surface of $\mathrm{Li}$ electrode, and charge transfer resistance, respectively; and $\mathrm{CPE}_{\mathrm{SEl}}$ and $\mathrm{CPE}_{\mathrm{dl}}$ denote the corresponding constant phase elements. 
(a)

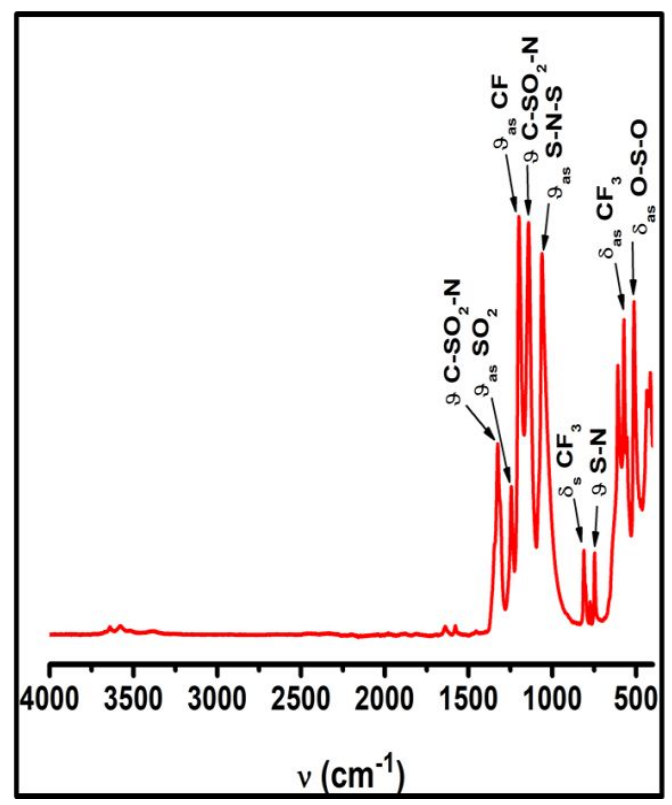

(b)

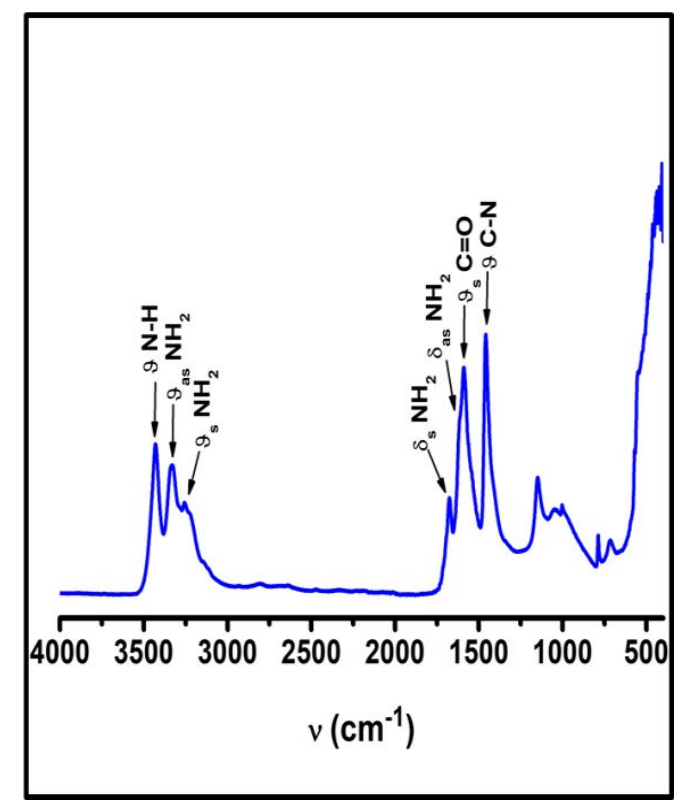

Figure S3. Fourier-transform infrared spectra of (a) pure bis(trifluoromethane sulfonyl)imide lithium salt and (b) urea. 


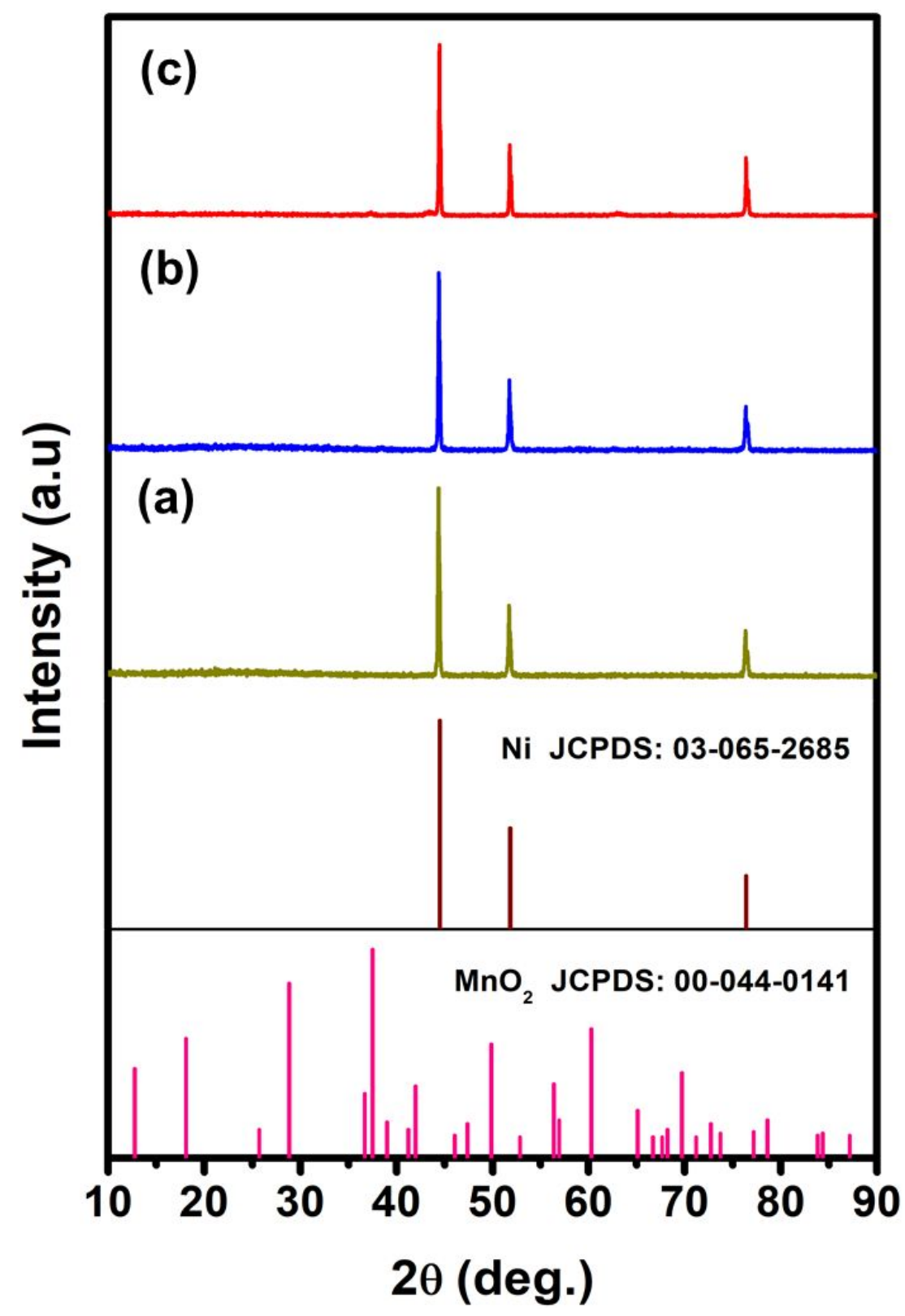

Figure S4. X-ray diffraction patterns of $\mathrm{MnO}_{2}$ catalyst formed on $\mathrm{Ni}$ foam: (a) fresh $\mathrm{Ni}$ foam, (b) before annealing, and (c) after annealing at $450{ }^{\circ} \mathrm{C}$ for $5 \mathrm{~h}$. 


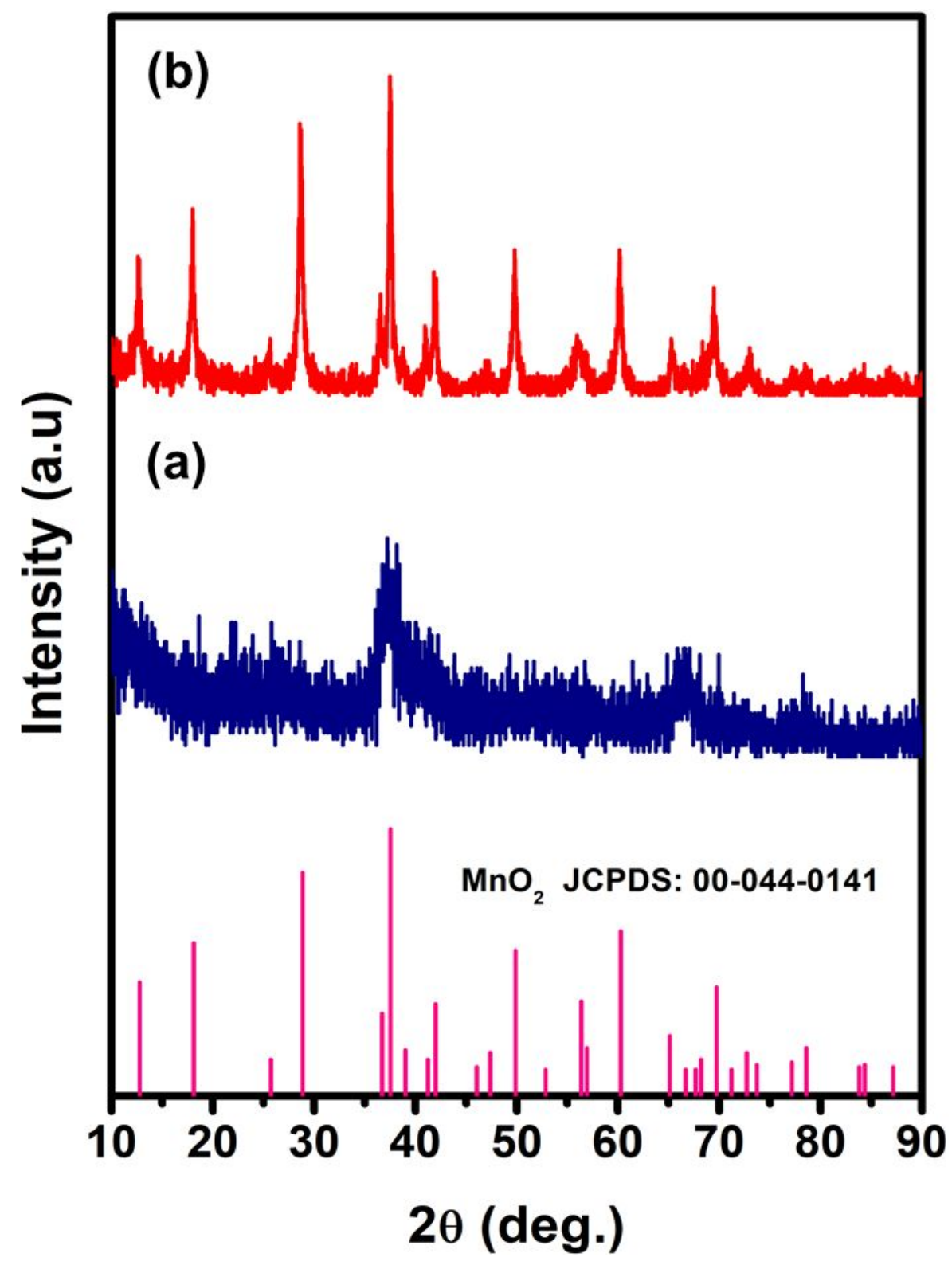

Figure S5. X-ray diffraction patterns of $\mathrm{MnO}_{2}$ powder obtained via hydrothermal method:

(a) before annealing, and (b) after annealing at $450^{\circ} \mathrm{C}$ for $5 \mathrm{~h}$. 

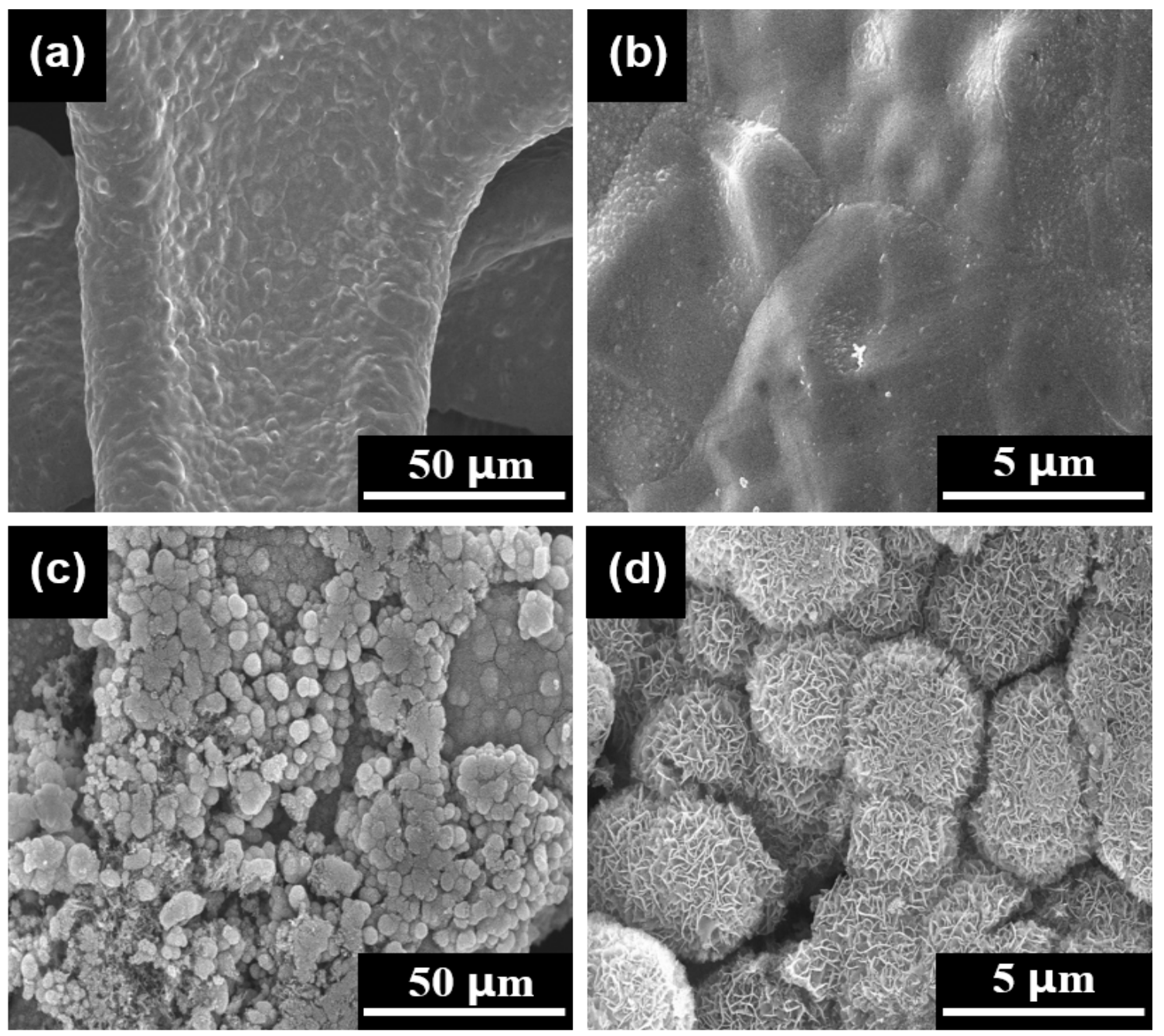

Figure S6. (a) Low- and (b) high-magnification scanning electron microscopy (SEM) images of fresh $\mathrm{Ni}$ foam. (c) Low- and (d) high-magnification SEM images of $\mathrm{MnO}_{2}$ catalyst formed on $\mathrm{Ni}$ foam. 

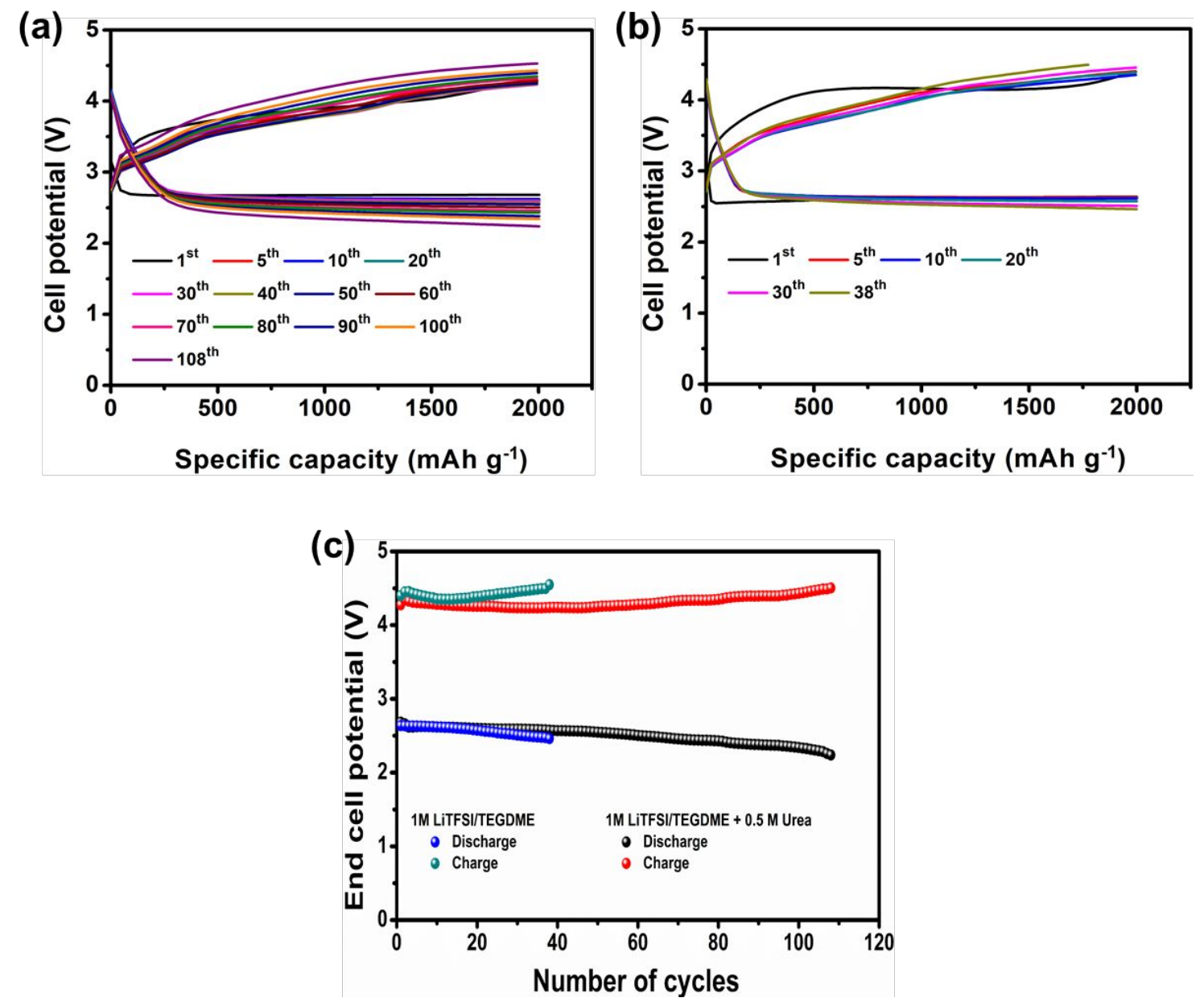

Figure S7. (a) and (b) Discharge-charge potential profiles of $\mathrm{Li}^{-} \mathrm{O}_{2}$ batteries with and without urea additive. (c) Variation of end cell potential with cycle number of $\mathrm{Li}-\mathrm{O}_{2}$ batteries with and without urea electrolyte additive. Measurements were carried out in limited capacity mode: $2000 \mathrm{mAh} \mathrm{g}{ }^{-1}$ and $0.2 \mathrm{~mA} \mathrm{~cm}{ }^{-2}$ in pure $\mathrm{O}_{2}$ atmosphere. Here, 
LiTFSI and TEGDME denote bis(trifluoromethane sulfonyl)imide lithium salt and tetraethylene glycol dimethyl ether, respectively. 